\title{
Antitumor actions of baicalein and wogonin in HT-29 human colorectal cancer cells
}

\author{
SO-JUNG KIM ${ }^{1}$, HYEONG-JIN KIM ${ }^{1}$, HYE-RI KIM ${ }^{1}$, SEUNG-HO LEE ${ }^{1}$, SUNG-DAE CHO ${ }^{2}$, \\ CHANG-SUN CHOI ${ }^{3}$, JEONG-SEOK NAM ${ }^{4}$ and JI-YOUN JUNG ${ }^{1}$ \\ ${ }^{1}$ Department of Companion and Laboratory Animal Science, Kongju National University, Yesan 340-702; \\ ${ }^{2}$ Department of Oral Pathology, School of Dentistry, Chonbuk National University, Jeonju 561-756; \\ ${ }^{3}$ Department of Food and Nutrition, College of Human Ecology, Chung-Ang University, Ansung 456-756; \\ ${ }^{4}$ Lee Gil Ya Cancer and Diabetes Institute, Gachon University of Medicine and Science, Inchon 406-840, Republic of Korea
}

Received April 18, 2012; Accepted September 11, 2012

DOI: $10.3892 / \mathrm{mmr} .2012 .1085$

\begin{abstract}
The purpose of this study was to determine the effects of baicalein and wogonin, which are compounds derived from the Chinese herb Scutellaria baicalensis, in suppressing the viability of HT-29 human colon cancer cells. Following treatment with baicalein or wogonin, several apoptotic events were observed, including DNA fragmentation, chromatin condensation and increased cell cycle arrest in the G1 phase. Baicalein and wogonin decreased Bcl-2 expression, whereas the expression of Bax was increased in a dose-dependent manner compared with the control. Furthermore, the induction of apoptosis was accompanied by an inactivation of phosphatidylinositol 3-kinase (PI3K)/Akt in a dose-dependent manner. The administration of baicalein to mice resulted in the inhibition of the growth of HT-29 xenografts without any toxicity following 5 weeks of treatment. The results indicated that baicalein induced apoptosis via Akt activation in a p53-dependent manner in the HT-29 colon cancer cells and that it may serve as a chemopreventive or therapeutic agent for HT-29 colon cancer.
\end{abstract}

\section{Introduction}

Colorectal cancer is a growing health problem. It is a leading cause of cancer mortality in men and women and affects more than one million people annually worldwide (1). The features of the disease usually occur progressively over a protracted period due to increased genomic instability, leading to the upregulation of oncogenes and the downregulation of tumor suppressor genes (2). Studies have shown that major intracellular signaling pathways are altered during tumorigenesis,

Correspondence to: Professor Ji-Youn Jung, Department of Companion and Laboratory Animal Science, Kongju National University, Daehoe-ri, Yesan-eup, Yesan-gun, Chungcheongnam-do, Republic of Korea

E-mail: wangza@kongju.ac.kr

Key words: apoptosis, baicalein, colon cancer, flavonoid, HT-29, wogonin leading to the dysregulation of processes such as proliferation and survival (3).

The induction of apoptosis in tumor cells is a strategy used in antitumor therapy. The balance between survival- and apoptosis-signaling pathways controls tumor pathogenesis. A number of flavonoids exert potent antitumor activity through the induction of apoptosis and cell cycle arrest in several tumor cell lines $(4,5)$. Flavonoids, a group of polyphenolic compounds, are natural products present in numerous fruits and vegetables and in all vascular plants (6). The antitumor activity of flavonoids has been the subject of much attention.

Scutellaria baicalensis, known as Chinese skullcap and Huang Qin, contains several flavonoids and is a widely used herb in traditional Chinese medicine with antitumor, antiviral, antibacterial and anti-inflammatory properties (7-11). Baicalein and wogonin are flavonoid-like chemical compounds which are found in Scutellaria baicalensis and have been reported to inhibit cell growth and induce apoptosis in various tumors $(12,13)$. However, the precise mechanisms by which baicalein and wogonin induce apoptosis are not yet known.

We compared the antitumor efficacies and mechanisms of action of baicalein and wogonin in HT-29 human colorectal tumor cells. Additionally, a mouse xenograft model was used to evaluate the antitumor activities of baicalein and wogonin in vivo. Understanding the underlying mechanisms of baicaleinand wogonin-induced apoptosis may benefit the development of chemopreventives and chemotherapeutics for colon tumors.

\section{Materials and methods}

Cell culture and reagents. The HT-29 cells were obtained from the Korean Cell Line Bank (KCLB, Korea) and were cultured in RPMI-1640 medium supplemented with $10 \%$ fetal bovine serum (FBS) and $1 \%$ penicillin/streptomycin. The cells were subsequently incubated for $2 \mathrm{~h}$ at $37^{\circ} \mathrm{C}$ in an atmosphere of $5 \% \mathrm{CO}_{2}$. Wogonin was purchased from Wako (Osaka, Japan). Baicalein, MTT [3-(4,5-dimethylthiazol2-yl)-2,5-diphenyl tetrazolium bromide], dimethylsulfoxide (DMSO) and propidium iodide (PI) were purchased from Sigma-Aldrich (St. Louis, MO, USA). The RPMI-1640 medium, penicillin/streptomycin and trypsin-EDTA were 
obtained from Hyclone Laboratories, Inc. (Logan, UT, USA). FBS was obtained from Gibco-BRL (Grand Island, NY, USA). Anti-Akt, anti-phospho-Akt (Ser473), anti-Bcl-2, anti-Bax, anti-p53, anti-p21, anti-cleaved-poly(ADP-ribose) polymerase (PARP), anti-phospho-GSK-3 $\beta$, anti-cyclin-B1, anti-survivin and anti- $\beta$-actin were purchased from Cell Signaling Technology, Inc. (Beverly, MA, USA). Anti-cyclin D1 (HD11) was purchased from Santa Cruz Biotechnology, Inc. (Santa Cruz, CA, USA). Cell lysis buffer and 4',6-diamidino2-phenylindole (DAPI) were obtained from Invitrogen Life Technologies (Carlsbad, CA, USA). The fluorescein isothiocyanate (FITC)-conjugated annexin $\mathrm{V}$ apoptosis detection kit was purchased from BD Biosciences (San Diego, CA, USA).

Cell viability assessment. Cells were seeded in 12-well plates at $5 \times 10^{4}$ cells/well $(24 \mathrm{~h})$ and $4 \times 10^{4}$ cells/well $(48 \mathrm{~h})$ and then incubated with either baicalein or wogonin at 0, 25, 50 and $100 \mu \mathrm{M}$ for the indicated times. The medium was removed and the cells were incubated with $1000 \mu \mathrm{l}$ MTT solution $(2 \mathrm{mg} / \mathrm{ml}$ MTT in PBS) for $4 \mathrm{~h}$. The optical densities of the solutions were determined using a spectrophotometer (Ultrospec 2100 pro; Amersham Biosciences, Piscataway, NJ, USA) at $540 \mathrm{~nm}$. The cell viability was expressed as the optical density ratio of the treated cells to the control.

Nuclear morphology. Cells were seeded in 12-well plates at $4 \times 10^{4}$ cells/well and then incubated with either baicalein or wogonin at $0,25,50$ and $100 \mu \mathrm{M}$ for $48 \mathrm{~h}$. Following treatment, the cells were fixed with $4 \%$ paraformaldehyde in PBS for $30 \mathrm{~min}$ in an incubator. The fixed cells were washed twice with PBS and the cell nuclei were stained with DAPI in PBS. Specific fluorescence was observed using a fluorescence microscope (Bx-41; Olympus, Tokyo, Japan).

Annexin $V$ staining for apoptosis analysis. Adherent and floating HT-29 cells grown in $75-\mathrm{cm}^{2}$ flasks were collected following mild trypsinization. The trypsinized cells were washed once with PBS, then resuspended in $100 \mu \mathrm{l}$ annexin $\mathrm{V}$ binding buffer and mixed with FITC-conjugated annexin V and phycoerythrin (PE)-conjugated PI. The resuspended cells were incubated at room temperature in the dark for $15 \mathrm{~min}$. Apoptotic cells (annexin V + PI) were measured within $1 \mathrm{~h}$ using a flow cytometer (FACSCalibur; BD Biosciences).

Flow cytometric analysis of the cell cycle. HT-29 cells $\left(1.5-2 \times 10^{5}\right)$ were seeded in $25-\mathrm{cm}^{2}$ flasks. After $24 \mathrm{~h}$, the medium was replaced with fresh medium (control) or fresh medium supplemented with baicalein or wogonin. After 2 days of incubation (at 50-60\% cell confluence), the cells were trypsinized, collected and centrifuged for $5 \mathrm{~min}$ at 1,700 rpm. The cell pellets were washed twice with PBS and then fixed with $70 \%$ ethanol for $30 \mathrm{~min}$. DNA fragments were then stained with $50 \mu \mathrm{g} / \mathrm{ml} \mathrm{PI}$ and $100 \mu \mathrm{g} / \mathrm{ml}$ of RNase (Sigma-Aldrich) in PBS for $30 \mathrm{~min}$ at room temperature. The viable cells were sorted and the fluorescence intensity was measured by flow cytometry.

Western blotting. Briefly, floating cells were collected and protein concentrations were measured using the Bradford assay (14). Equal amounts of protein were separated by sodium dodecyl sulfate-polyacrylamide gel electrophoresis and then electrophoretically transferred onto nitrocellulose membranes (Bio-Rad Laboratories, Inc., Hercules, CA, USA). The transferred membranes were blocked with Tris-buffered saline containing 5\% non-fat dry milk and $0.1 \%$ Tween-20 for $2 \mathrm{~h}$ at $4^{\circ} \mathrm{C}$.

The blocked membranes were incubated with primary antibodies overnight at $4^{\circ} \mathrm{C}$ with gentle agitation. The membranes were then incubated with horseradish peroxidase-conjugated goat anti-mouse and anti-rabbit IgG secondary antibodies for $1 \mathrm{~h}$ at room temperature with gentle agitation. After washing, the bands were visualized using enhanced chemiluminescence (ECL) detection reagents (Pierce Biotechnology, Inc., Rockford, IL, USA).

Nude mouse xenograft assay. Male nude mice (6 weeks old) were purchased from Orient Ltd. (Seoul, Korea). Xenografts were established by subcutaneous injection of in vitro-cultured HT-29 cells $\left(10^{6}\right.$ cells $\left./ 200 \mu \mathrm{l}\right)$ into the flanks of donor nude mice. All procedures involving the use of the animals were approved by the Institutional Animal Care and Use Committee of Kong-Ju National University and were carried out in accordance with the ethical guidelines.

When the tumors reached $\sim 1000 \mathrm{~mm}^{3}$ in size, the mice were anesthetized with diethyl ether and the tumor masses were obtained surgically from the mice. The masses were sliced into $2 \times 2 \mathrm{~mm}$ fragments using a grid and the tumor fragments were implanted surgically into the subcutaneous tissue of the right flank of each mouse (15). The mice were randomized into three groups each comprising four mice (two tumors/mouse). In the treatment groups, animals were treated orally with baicalein or wogonin $(10 \mathrm{mg} / \mathrm{kg})$ three times/week for 43 days starting on the day of tumor cell implantation. The control mice received an equal volume of the vehicle. The tumors were measured in two diameters with calipers to permit calculation of the tumor volume using the formula: $\mathrm{V}=\{(\mathrm{D}+\mathrm{d}) / 2\}^{3}$, where $\mathrm{D}$ and $\mathrm{d}$ are the larger and smaller diameters, respectively. The statistical significance of differences in tumor volume, wet tumor weight and body weight between the control and treated mice were assessed by the Student's t-test.

Preparation and administration of baicalein and wogonin. Groups of four mice were randomly assigned to receive one of the treatments starting on the day of implantation of the tumor fragment. In the control group, each mouse was administered corn oil ( $0.2 \mathrm{ml} /$ day $)$ every day for 5 weeks. In the treatment groups, each mouse was administered $10 \mathrm{mg} / \mathrm{kg}$ baicalein or wogonin every day for 5 weeks.

Immunohistochemistry. Briefly, $4-\mu$ m-thick sections were cut from paraffin-embedded tissue blocks. The sections were deparaffinized and hydrated by sequential immersion in xylene and graded alcohol solutions. The endogenous peroxidase activity was quenched by treatment with $3 \%$ hydrogen peroxide for $5 \mathrm{~min}$ at room temperature. The sections were incubated with primary antibodies for $1 \mathrm{~h}$ at $37^{\circ} \mathrm{C}$ and then with the secondary antibody for $30 \mathrm{~min}$ at room temperature. Staining was performed using diaminobenzidine (DAB) and counterstaining was performed using methyl green. For the negative control, the incubated antibody diluent was used as a substitute for the primary antibody. 
A<smiles>Oc1cc2c(c(O)c1O)C(O)C=C(c1ccccc1)O2</smiles>

Baicalein $(\mu \mathrm{M})$<smiles>COc1c(O)cc(O)c2c1OC(c1ccccc1)=CC2O</smiles>

Wogonin $(\mu \mathrm{M})$
C

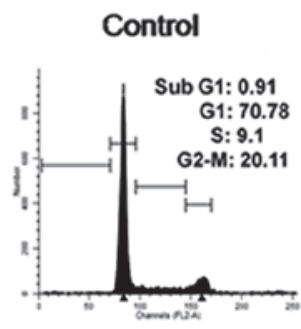

B
$24 \mathrm{~h}$

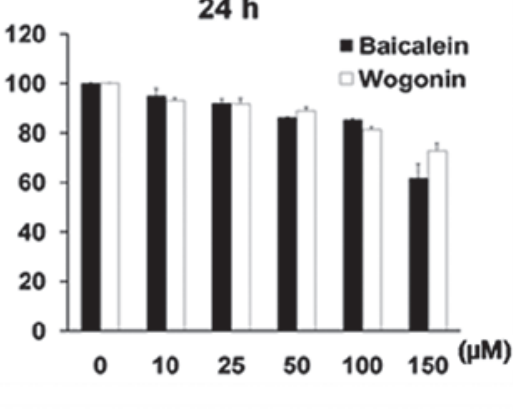

$48 h$

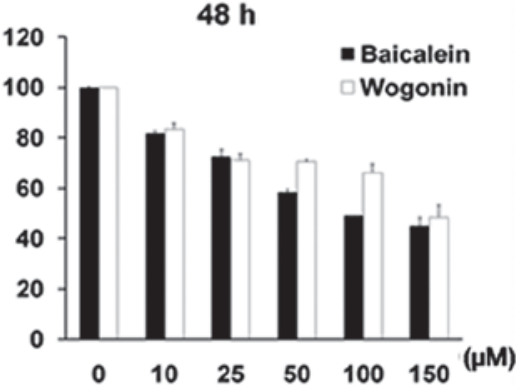

Figure 1. Effects on cell growth and cell cycle progression. (A) Chemical structures of baicalein $\left(\mathrm{C}_{15} \mathrm{H}_{10} \mathrm{O}_{5} ; \mathrm{MW}\right.$ : 270.24) and wogonin $\left(\mathrm{C}_{16} \mathrm{H}_{12} \mathrm{O}_{5}\right.$; MW: 284.26). (B) Attenuation of HT-29 colon tumor cell growth by baicalein and wogonin, assessed by the MTT assay. Each point represents three experiments, each carried out in triplicate. (C) Cell cycle analysis of baicalein- and wogonin-treated cells. Cells were treated with vehicle (DMSO) or with the indicated concentrations of baicalein or wogonin for 2 days. Data shown are representative of three experiments showing similar results. MTT, 3-(4,5-dimethylthiazol2-yl)-2,5-diphenyl tetrazolium bromide; DMSO, dimethylsulfoxide.

Apoptotic cell detection using the terminal deoxynucleotidyl transferase dUTP nick end labeling (TUNEL) assay. Tumor tissues were examined using the Dead End ${ }^{\mathrm{TM}}$ Colorimetric TUNEL System (Promega Corporation, Madison, WI, USA). After dewaxing and rehydration, the sections were treated with proteinase $\mathrm{K}(20 \mu \mathrm{g} / \mathrm{ml})$ for $15 \mathrm{~min}$ at room temperature. Endogenous peroxidase was blocked with $0.3 \%$ hydrogen peroxide in PBS for $5 \mathrm{~min}$. Digoxigenin dUTP end-labeled DNA was detected with anti-digoxigenin peroxidase, followed by peroxidase detection with $0.05 \%$ DAB containing $0.02 \%$ hydrogen peroxide. Tissue sections were counterstained with methyl green. Three randomly selected fields in each tumor section were counted for apoptotic bodies.

Statistical analysis. All data are expressed as the mean \pm SE (standard error). One-way ANOVA was used to analyze statistical differences among multiple comparisons. $\mathrm{P}<0.05$ was considered to indicate a statistically significant result.

\section{Results}

Cell growth inhibition assessed by the colorimetric MTT assay. The chemical structures of baicalein and wogonin are shown in Fig. 1A. The growth inhibitory effects of the two agents on the HT-29 cells were determined as the percentage of viable cells in the treated cells compared with the untreated control. As shown in Fig. 1B, treatment with 10-150 $\mu \mathrm{M}$ baicalein or wogonin for 24 or $48 \mathrm{~h}$ resulted in a slight decrease in the cell viability of the HT-29 cells. Baicalein and wogonin demonstrated significant cytotoxicity after $48 \mathrm{~h}$. In the 24-h and 48-h treatment groups, wogonin $(150 \mu \mathrm{M})$ reduced cell viability by 27.28 and $51.66 \%$, respectively. Baicalein $(100 \mu \mathrm{M})$ reduced cell viability by more than $50 \%$ in the 48 -h treatment group.

Effects of baicalein and wogonin on cell cycle arrest and induction of apoptosis in HT-29 cells. The results shown in Fig. 1C indicate that baicalein and wogonin induced sub-G1 and $\mathrm{G} 2 / \mathrm{M}$ arrest. In the cells treated with baicalein, when the drug concentration was increased from 25 to $100 \mu \mathrm{M}$, the percentage of cells in the sub-G1 phase increased from 0.8 to $15.45 \%$ and the percentage of cells in the G2/M phase (apoptotic fraction) increased from 14.87 to $36.08 \%$. However, the $\mathrm{S}$ phase was not markedly altered by baicalein.

In the cells treated with wogonin, when the drug concentration was increased from 25 to $100 \mu \mathrm{M}$, the percentage of cells in the sub-G1 phase increased from 0.62 to $9.57 \%$ and the 

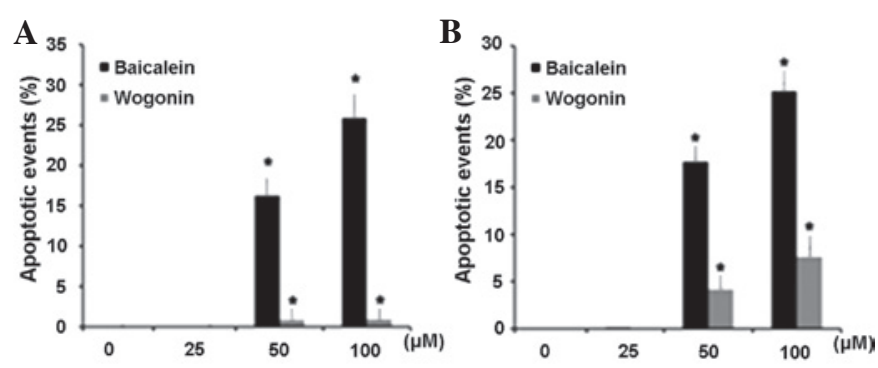

C

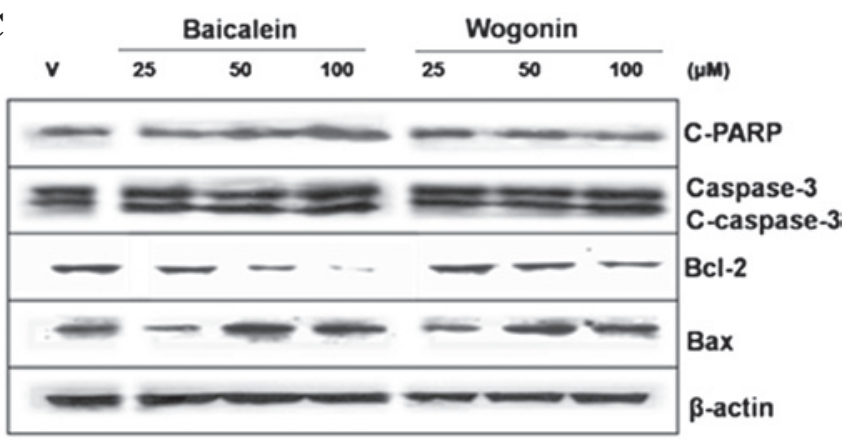

Figure 2. Induction of apoptosis in HT-29 cells by treatment with baicalein or wogonin for $48 \mathrm{~h}$. (A) Quantified results of DAPI staining. Six high-power fields per coverslip were analyzed. For each condition, experiments were performed in duplicate. The data shown are representative of four individual experiments. The results are presented as the means $\pm \mathrm{SD}(\mathrm{p}<0.001)$ for the comparison of the control group. (B) Quantified results of annexin V/PI double staining. Apoptotic events were calculated as described in Materials and methods. ${ }^{*} \mathrm{P}<0.05$. In $\mathrm{A}$ and $\mathrm{B}$, the results are the average of three independent experiments with similar results. (C) Immunoblotting for Bcl-2 family members, caspase-3 and PARP. DAPI, 4',6-diamidino-2-phenylindole; PI, propidium iodide; PARP, poly(ADP-ribose) polymerase; $\mathrm{C}$, cleaved.

percentage of cells in the G2/M phase increased from 16.61 to $27.46 \%$. No significant change in the $\mathrm{S}$ phase was observed following wogonin treatment in our experiments.

Apoptosis assessment by DAPI staining and annexin V and PI double staining. The quantified results of the DAPI staining are presented in Fig. 2A. A dose-dependent increase was observed in the number of HT-29 cells showing nuclear condensation and fragmentation following treatment with either baicalein or wogonin $(25,50$ and $100 \mu \mathrm{M})$ for $48 \mathrm{~h}$ (Fig. 2A). Treatment of the HT-29 cells with wogonin for $48 \mathrm{~h}$ significantly increased the percentage of apoptotic cells from $0.11 \%$ in the control group to $0.12 \%(25 \mu \mathrm{M}), 0.88 \%(50 \mu \mathrm{M})$ and $0.93 \%(100 \mu \mathrm{M})$. Treatment of the HT-29 cells with baicalein for $48 \mathrm{~h}$ significantly increased the percentage of apoptotic cells from $0.11 \%$ in the control group to $16.27 \%(50 \mu \mathrm{M})$ and $25.92 \%(100 \mu \mathrm{M})$.

Additionally, flow cytometric analysis with annexin V/PI double staining was used to determine the levels of apoptosis elicited by baicalein and wogonin. The percentages of apoptotic cells induced by 25,50 and $100 \mu \mathrm{M}$ baicalein were $0.15,17.74$ and $25.22 \%$, respectively, whereas only $0.01 \%$ of the control cells were apoptotic (Fig. 2B). The results clearly indicate that baicalein induced apoptosis in the HT-29 cells.

Intracellular signaling in baicalein- and wogonin-induced apoptosis. To obtain further evidence of the apoptosis-inducing effect of baicalein in HT-29 cells, the expression levels of caspase-3, which is the key indicator of intracellular signaling

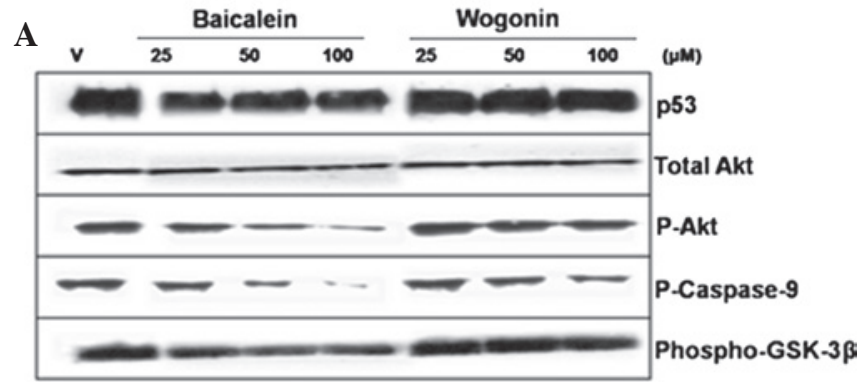

B

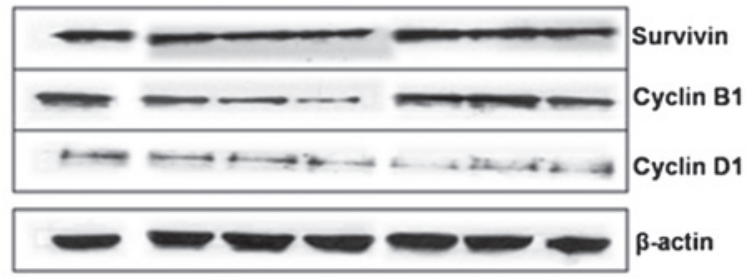

Figure 3. Downregulation of Akt-mediated signaling pathways by treatment with baicalein and wogonin for $48 \mathrm{~h}$. (A) Activity of Akt and its downstream effectors as assessed by immunoblotting. (B) Survivin, cyclin B1 and cyclin D1 expression quantified by immunoblotting. P, phospho.

in apoptosis, were determined. Cleaved caspase-3 levels were increased following treatment with 25,50 and $100 \mu \mathrm{M}$ baicalein and $100 \mu \mathrm{M}$ wogonin for $48 \mathrm{~h}$ (Fig. 2C).

We also investigated whether the apoptosis was linked to the expression of the Bcl-2 (anti-apoptotic) and Bax (pro-apoptotic) proteins, which are central regulators of apoptosis. Following incubation with $25-100 \mu \mathrm{M}$ baicalein or wogonin for $48 \mathrm{~h}$, the expression level of Bcl-2 decreased markedly, whereas that of Bax increased significantly. Additionally, baicalein induced a significant increase in PARP cleavage compared with the control group (Fig. 2C). To assess the involvement of the Akt pathway in the apoptosis, the levels of phosphorylated Akt protein were investigated by western blotting. The amount of active phosphorylated Akt was clearly reduced by the baicalein treatment while the total Akt expression level was unchanged (Fig. 3). As observed in Fig. 3A, the p53 protein level was reduced by the baicalein treatment. Since phospho-GSK-3 $\beta$ is another downstream effector of Akt, we also investigated the phospho-GSK-3 $\beta$ expression levels by western blotting. As shown in Fig. 3A, the phospho-GSK-3 $\beta$ expression level was markedly downregulated by the baicalein treatment, consistent with the inhibition of Akt phosphorylation following treatment. Also, the baicalein and wogonin treatments clearly reduced phospho-caspase-9 levels.

As shown in Fig. 3B, the protein levels of cyclin D1 and cyclin B1, as assessed by immunoblotting, were reduced in a concentration-dependent manner (Fig. 3B). The amount of survivin was also reduced by the baicalein treatment (Fig. 3B).

Inhibition of colon tumor growth by baicalein and wogonin in xenograft models. Next, we investigated the effects of baicalein and wogonin on colon tumor cells in vivo. Our results revealed that significant tumor growth inhibition occurred in the baicalein-treated mice compared with the untreated mice (Fig. 4A). Additionally, baicalein was shown to significantly 
A

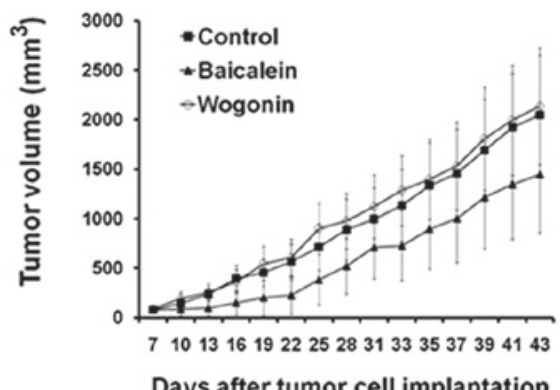

C

TUNEL
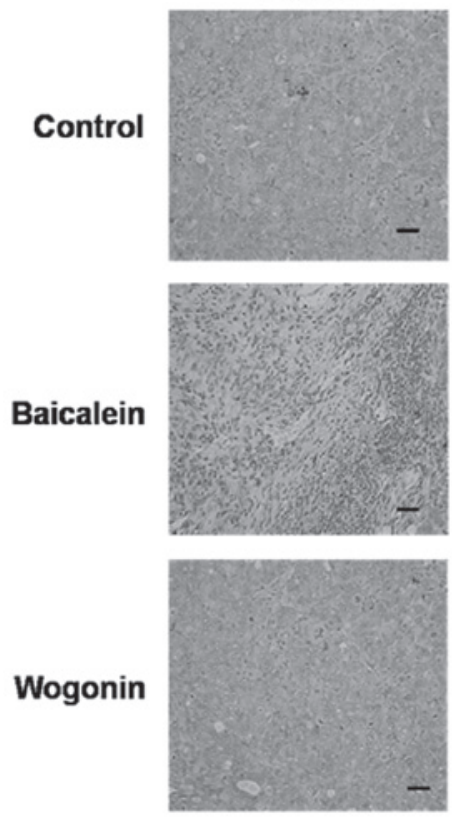

B

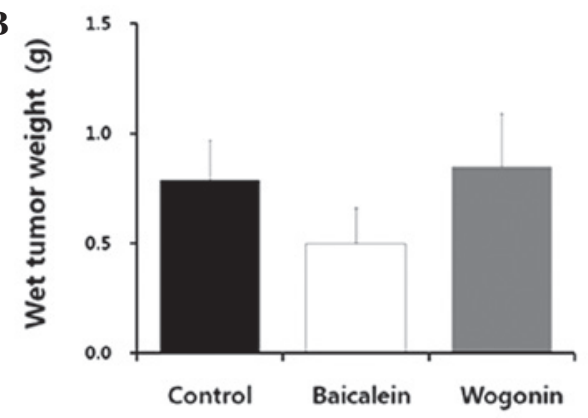

P21
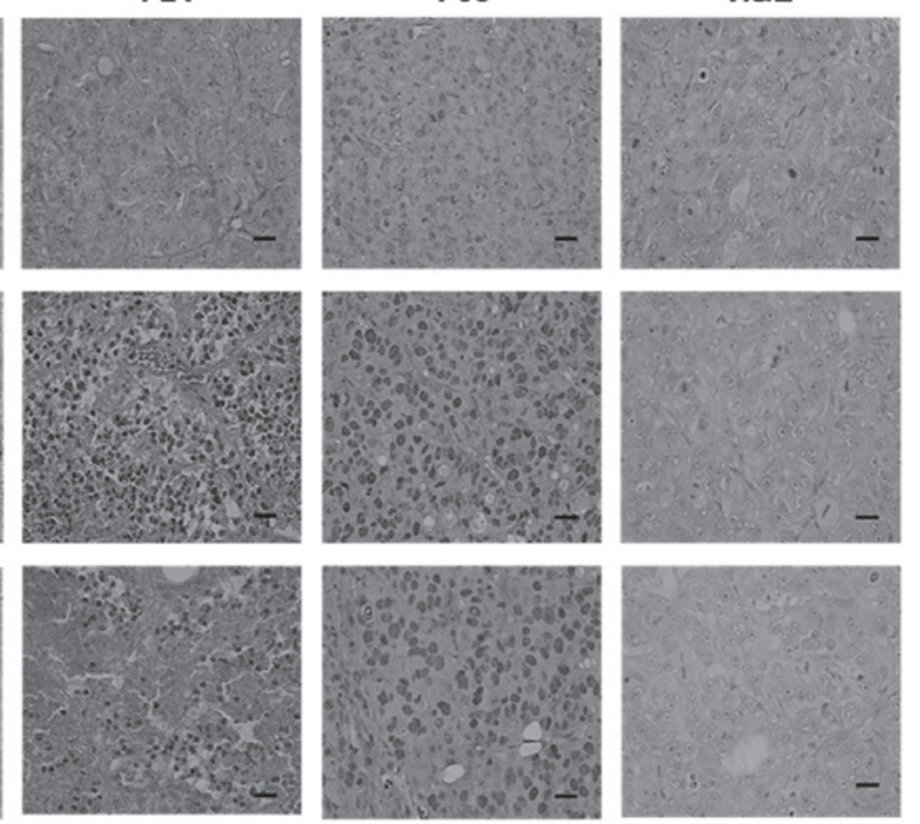

Figure 4. Inhibition of colon tumor growth by baicalein and wogonin in human colon tumor xenografts. Xenograft tumor $($ A $)$ volume and $(B)$ weight. Bars = SE (C) Baicalein and wogonin induced p21-, p53- and TUNEL-positive cells as well as H\&E-stained apoptotic cells as assessed by immunohistochemistry. Slides were observed under a microscope and images captured (x400). Scale bar $=20 \mu \mathrm{m}$. TUNEL, terminal deoxynucleotidyl transferase dUTP nick end labeling; $\mathrm{H} \& \mathrm{E}$, hematoxylin and eosin.

decrease tumor weights (Fig. 4B). No significant difference in tumor weight was observed between the wogonin-treated and control groups.

The average tumor volumes following 5 weeks of treatment were $1,448.60 \mathrm{~mm}^{3}$ (baicalein; $10 \mathrm{mg} / \mathrm{kg} /$ day), $2,137.85 \mathrm{~mm}^{3}$ (wogonin; $10 \mathrm{mg} / \mathrm{kg} /$ day) and $2,049.85 \mathrm{~mm}^{3}$ (control) (Fig. 4A). The inhibition rates for baicalein and wogonin were 29.33 and $-4.29 \%$, respectively (Table I). Baicalein inhibited the colorectal tumor growth to $87 \%$ of control values $(n=4$; $\mathrm{P}<0.05$ ), whereas the colorectal tumor growth following wogonin treatment was not significantly different from that of the controls. The difference in trends was statistically significant $(\mathrm{P}<0.001)$. The average tumor volumes are shown in Table I and reveal that baicalein suppressed colon tumor growth in the xenograft model.

Additionally, in the mice treated with $10 \mathrm{mg} / \mathrm{kg}$ baicalein or wogonin, when body weight, organ histology and kidney function were compared with those of the controls, no evidence of drug-related toxicity was observed (data not shown).

Effects of baicalein and wogonin on p21 and p53 levels in HT-29 cells. A TUNEL assay was performed on the tumor tissues from the experimental animals. A marked increase in the number of apoptotic cells in the baicalein-treated HT-29 tumor xenograft tissue was observed, as indicated by the dark brown nuclear staining of the apoptotic cells. However, no significant induction of apoptosis was observed in the wogonin-treated cells (Fig. 4C). These in vivo results support the in vitro findings that baicalein had an apoptotic effect in HT-29 cells.

The morphological aspects of the apoptosis were studied immunohistochemically in the HT-29 cells following treatment with baicalein and wogonin. Increases in the $\mathrm{p} 53$ protein levels were detected in the baicalein- and wogonin-treated HT-29 cells. Although p21 protein is rarely observed in the wogonin-treated and control tumor tissues, it was definitely identified in the baicalein-treated tissues. These data indicate that increased levels of p53 and p21 are involved in baicaleininduced apoptosis, whereas only a slight induction of p21 was detected in the wogonin-treated cells (Fig. 4C).

Morphological analysis of hematoxylin and eosin-stained tissue sections was also performed (Fig. 4C). However, only a few mitotic nuclei cells were observed following the baicalein and wogonin treatments. 
Table I. Tumor inhibition rate of mice implanted with colon cancer cells treated with wogonin and baicalein.

\begin{tabular}{lcccccc}
\hline & \multicolumn{2}{c}{ Pre-experiment } & & \multicolumn{2}{c}{ Post-experiment } & \\
\cline { 2 - 3 } Group & $\mathrm{n}$ & Size $\left(\mathrm{mm}^{3}\right)$ & & $\mathrm{n}$ & Size $\left(\mathrm{mm}^{3}\right)$ & $\begin{array}{c}\text { Inhibition } \\
\text { rate }(\%)\end{array}$ \\
\hline Control & 4 & 80.3 & & 4 & 2049.9 & \\
Baicalein & 4 & 81.2 & & 4 & 1448.6 & 29.33 \\
Wogonin & 4 & 92.6 & & 4 & 2137.9 & -4.29 \\
\hline
\end{tabular}

\section{Discussion}

We evaluated and compared the antitumor effects of baicalein and wogonin, two compounds derived from S. baicalensis. The human colon tumor cell line HT-29 was cultured in the absence and presence of varying concentrations of baicalein and wogonin. Moreover, to understand whether the baicaleinand wogonin-induced inhibition of cell viability was due to cell cycle arrest, PI staining was performed.

Flavonoid treatment resulting in histone hyperacetylation leads to manifold effects in tumor cells, including the modulation of gene expression and cell death, and occurs to varying extents in different cell types. As shown in Fig. 1, wogonin induced cell death in HT-29 cells with a higher percentage of necrosis than apoptosis. Since PARP causes ATP depletion, it appears plausible that its inhibition favors apoptosis over necrosis, as apoptosis is an ATP-dependent, energy-consuming process (16). Thus, the prevention of mitochondrial dysfunction and ATP depletion appear to be involved in switching the mode of wogonin-induced cell death in the HT-29 cells from a necrotic to a mainly apoptotic type. The primary trigger of wogonin-induced cell death, downstream of histone acetylation, remains to be identified.

Evidence suggests that the proteolytic degradation of specific substrates is responsible for many of the morphologic and biochemical features of apoptosis. Increased levels of nuclear fragmentation and total apoptosis were observed in the cells by DAPI staining (Fig. 2A) and annexin V/PI double staining (Fig. 2B), respectively. The assays demonstrated that baicalein significantly inhibited cell growth and induced apoptosis more potently than wogonin in the HT-29 cells (Fig. 2A and B).

Previous studies have demonstrated that $\mathrm{Bcl}-2$ protein family members significantly regulate apoptosis, either as activators (e.g. Bax) or as inhibitors (e.g. Bcl-2) (17,18). In the present study, we identified that baicalein and wogonin treatment significantly induced mitochondrial damage and apoptosis, modulated Bcl-2 and Bax protein expression and led to mitochondrial dysfunction, caspase activation and PARP cleavage in HT-29 cells (Fig. 2C). Our data demonstrate that baicalein- and wogonin-induced apoptosis is related to increased levels of Bax and decreased levels of Bcl-2 and also the induction of mitochondrial dysfunction, leading to the apoptosis of the HT-29 cells.

Caspase-3 is one of the key executioners of apoptosis, as it is either partially or completely responsible for the proteolytic cleavage of a number of key proteins, including PARP. PARP is critical for cell viability. The cleavage of PARP facilitates cellular disassembly and serves as a marker of cells undergoing apoptosis (16). Our in vitro results in HT-29 cells indicate the concurrent occurrence of apoptotic DNA fragmentation, caspase- 3 activation and PARP cleavage in the baicalein-treated HT-29 cells (Fig. 2C). These results indicate that the induction of apoptosis by baicalein is mediated by a p53-dependent or p53-independent pathway followed by the stimulation of the activities of caspase-3 and endonuclease. The cross-linking between the p53-dependent and p53-independent pathways in the baicalein- and wogonin-induced apoptosis remains unclear.

The phosphatidylinositol 3-kinase (PI3K)/Akt pathway is associated with cell survival through the activation of anti-apoptotic downstream effectors $(19,20)$. Therefore, we investigated the expression of phosphorylated caspase-9, a downstream target of Akt, and found that the baicalein treatment significantly reduced the levels of phospho-caspase-9 (Ser196). These data suggest that the baicalein-induced apoptosis was caspase-9-dependent. Also, changes in the levels of phospho-Akt, phospho-GSK-3 $\beta$, cyclin B1 and cyclin D1 suggest that the Akt-mediated canonical Wnt-signaling pathway was downregulated by baicalein in the colon tumor cells (Fig. 3A and B). Inhibition of the Akt pathway downregulates survivin expression and enhances apoptosis in tumor cells (21). Thus, baicalein elicits activation of Akt that may be caused by self-protection of tumor cells, which resist cell death by defending the survivin level. The blockade of the PI3K-Akt pathway by $\mathrm{PI} 3 \mathrm{~K}$ and Akt inactivation enhanced the reduction of survivin levels and promoted cytotoxicity in the baicaleinexposed cells.

We then investigated whether the growth-inhibitory activity observed with baicalein treatment in vitro would also occur in vivo. Our results demonstrated that tumor growth was significantly inhibited in the baicalein-treated mice compared with the untreated mice (Fig. 4A and B).

The in vivo experiments also served to examine the mechanism of the baicalein-induced apoptosis in the nude mice. Several studies have shown that the upregulation of p53 and p21 proteins results in the inhibition of molecules related to tumor cell growth and proliferation $(22,23)$. We identified that the levels of the apoptosis-related proteins induced by baicalein, particularly p53 and p21, were higher in the tissues of the baicalein- and wogonin-treated groups than those of the control group (Fig. 4C). Additionally, an increase in TUNEL-positive cells was found in the baicalein-treated group compared with the control group (Fig. 4C). These in vivo findings support the in vitro data and suggest that baicalein moderates apoptotic cell death in the HT-29 colon tumor xenografts through a p53-mediated apoptotic response.

Together, these results indicate that baicalein induces apoptosis in HT-29 cells via Akt inactivation and in a p53-dependent manner and that baicalein is a potential chemopreventive and therapeutic agent for colon tumors. These results should further contribute to the understanding of the antitumor activities of flavonoids.

\section{Acknowledgements}

This study was supported by a research grant from the Kongju National University in 2010. 


\section{References}

1. Jemal A, Murray T, Ward E, et al: Cancer statistics, 2005. CA Cancer J Clin 55: 10-30, 2005.

2. Samowitz WS and Slattery ML: Missense mismatch repair gene alterations, microsatellite instability, and hereditary nonpolyposis colorectal cancer. J Clin Oncol 20: 3178-3179, 2002.

3. Fang Q, Naidu KA, Naidu KA, et al: Ascorbyl stearate inhibits cell proliferation and tumor growth in human ovarian carcinoma cells by targeting the PI3K/AKT pathway. Anticancer Res 26: 203-209, 2006

4. Pan MH, Chen WJ, Lin-Shiau SY, Ho CT and Lin JK: Tangeretin induces cell-cycle G1 arrest through inhibiting Cyclin-dependent kinases 2 and 4 activities as well as elevating Cdk inhibitors p21 and p27 in human colorectal carcinoma cells. Carcinogenesis 23 1677-1684, 2002.

5. Hirano T, Abe K, Gotoh M and Oka K: Citrus flavone tangeretin inhibits leukaemic HL-60 cell growth partially through induction of apoptosis with less cytotoxicity on normal nymphocytes. Br J Cancer 72: 1380-1388, 1995.

6. Di Carlo G, Mascolo N, Izzo AA and Capasso F: Flavonoids: old and new aspects of a class of natural therapeutic drugs. Life Sci 65: 337-353, 1999

7. Razina TG, Udintsev SN, Tiutrin II, Borovskaia TG and Iaremenko KV: The role of thrombocyte aggregation function in the mechanism of the antimetastatic action of an extract of Baikal skullcap. Vopr Onkol 35: 331-335, 1989 (In Russian).

8. Konoshima T, Kokumai M, Kozuka M, et al: Studies on inhibitors of skin tumor promotion. XI. Inhibitory effects of flavonoids from Scutellaria baicalensis on Epstein-Barr virus activation and their anti-tumor-promoting activities. Chem Pharm Bull (Tokyo) 40: 531-533, 1992.

9. Kubo M, Kimura Y, Odani T, Tani T and Namba K: Studies on Scutellariae radix. Part II: The antibacterial substance. Planta Med 43: 194-201, 1981.

10. Kubo M, Matsuda H, Tanaka M, et al: Studies on Scutellariae radix. VII. Anti-arthritic and anti-inflammatory actions of methanolic extract and flavonoid components from Scutellariae radix. Chem Pharm Bull (Tokyo) 32: 2724-2729, 1984.

11. Mahmood N, Pizza C, Aquino R, et al: Inhibition of HIV infection by flavanoids. Antiviral Res 22: 189-199, 1993.

12. Kyo R, Nakahata N, Sakakibara I, Kubo M and Ohizumi Y: Baicalin and baicalein, constituents of an important medicinal plant, inhibit intracellular $\mathrm{Ca}^{2+}$ elevation by reducing phospholipase C activity in C6 rat glioma cells. J Pharm Pharmacol 50: 1179-1182, 1998.
13. Ma Z, Otsuyama K, Liu S, et al: Baicalein, a component of Scutellaria radix from Huang-Lian-Jie-Du-Tang (HLJDT), leads to suppression of proliferation and induction of apoptosis in human myeloma cells. Blood 105: 3312-3318, 2005.

14. Bradford MM: A rapid and sensitive method for the quantitation of microgram quantities of protein utilizing the principle of protein-dye binding. Anal Biochem 72: 248-254, 1976.

15. Datta SR, Brunet A and Greenberg ME: Cellular survival: a play in three Akts. Genes Dev 13: 2905-2927, 1999.

16. Zamaraeva MV, Sabirov RZ, Maeno E, Ando-Akatsuka Y, Bessonova SV and Okada Y: Cells die with increased cytosolic ATP during apoptosis: a bioluminescence study with intracellular luciferase. Cell Death Differ 12: 1390-1397, 2005.

17. Xiao D, Vogel V and Singh SV: Benzyl isothiocyanate-induced apoptosis in human breast cancer cells is initiated by reactive oxygen species and regulated by Bax and Bak. Mol Cancer Ther 5: 2931-2945, 2006.

18. Zhang M, Guo R, Zhai Y and Yang D: LIGHT sensitizes IFNgamma-mediated apoptosis of MDA-MB-231 breast cancer cells leading to down-regulation of anti-apoptosis Bcl-2 family members. Cancer Lett 195: 201-210, 2003.

19. Jin CY, Moon DO, Lee JD, et al: Sulforaphane sensitizes tumor necrosis factor-related apoptosis-inducing ligand-mediated apoptosis through down-regulation of ERK and Akt in lung adenocarcinoma A549 cells. Carcinogenesis 28: 1058-1066, 2007.

20. McDonald PC, Oloumi A, Mills J, et al: Rictor and integrin-linked kinase interact and regulate Akt phosphorylation and cancer cell survival. Cancer Res 68: 1618-1624, 2008.

21. Kim S, Kang J, Qiao J, Thomas RP, Evers BM and Chung DH: Phosphatidylinositol 3-kinase inhibition down-regulates survivin and facilitates TRAIL-mediated apoptosis in neuroblastomas. J Pediatr Surg 39: 516-521, 2004.

22. Su RY, Chao Y, Chen TY, Huang DY and Lin WW: 5-Aminoimidazole-4-carboxamide riboside sensitizes TRAILand TNF\{alpha\}-induced cytotoxicity in colon cancer cells through AMP-activated protein kinase signaling. Mol Cancer Ther 6: 1562-1571, 2007.

23. Hwang JT, Ha J, Park IJ, et al: Apoptotic effect of EGCG in HT-29 colon cancer cells via AMPK signal pathway. Cancer Lett 247: 115-121, 2007. 